AUGUST 1980

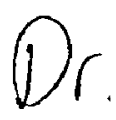

PPPL-1703

UC $-20 \mathrm{~g}$

\title{
MONTE CARLO EVALUATION OF TRANSPORT COEFFICIENTS
}

BY<smiles>[O]C1CC1</smiles>

A. H, BOOZER AND G, KUO-PETRAVIC

\section{PLASMA PHYSICS LABORATORY}

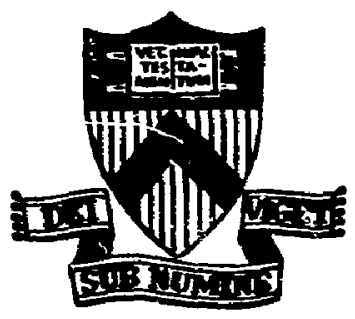

\section{PRINCETON UNIVERSITY PRINCETON, NEW JERSEY \\ HISTHBUTION DF THIS DOCLI!ERT IS URUMITES}

This work as suoported by the U.S. Dewarsment of Energy Contraet HO. DE-ACD2-7E-CHO 3073. Reprodustion, Eranslation, publication, use and dispesal, ir thole or in part. by or or the United Stetes government is permitico. 
Monte Carlo Evaluation of Transport Coefficients

Allen H. Boozer and Gioietta Kuo-Petravic

Princeton University, Plasma Physics Laboratory

Princeton, New Jersey 08544

A method is developed for evaluating transpori coefficients in asymetric geometries using the Monte Carlo methrd. The method is applied to the stellarator.

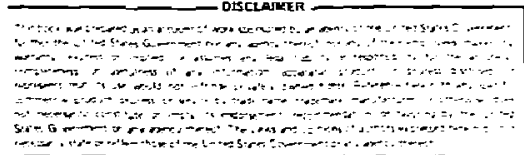




\section{INTRODUCTION}

Transport coefficients depend fundamentally on the number of symmetry directions of the plasma configuration. The enhancement of the transport coefficients in a torus over those in a straight circular cylinder is well known. 1-3 Actually, in the low collisionality banana regime, any breaking of the two symmetry directions of the straight circular cylinder gives a similar enhancement. That is, if the straight circular cylinder is distorted into an ellipse, made bumpy along its axis, twisted into a helix, ${ }^{4}$ or bent into a torus, the low collisionality transport is enhanced in much the same way. As long as one symmetry direction remains, this enhancement can be calculated with the techniques developed for the neoclassical theory of tokamaks. In addition, with at least one symnetry direction the magnetic field lines lie in well defined magnetic surfaces, which are also the constant pressuxe surfaces. In this paper, we will assume that good magnetic surfaces remain even in the absence of a symmetry direction. Conditions for this to be true have been discussed in the literature. ${ }^{5-9}$ In the absence of good surfaces, rapid electron parallel thermal conductivity precludes the usual magnetic fusion applications.

All experimental devices are asymmetric either due to the fundamental nature of the device, like the steliarator, or due to the lack of perfection in the realization of a concept, like a tokamak with toroidal ripple. In either case a very small breaking of the toroidal symmetry--by one percent or less--can enhance the ion thermal conductivity significantly over an equivalent symmetric device in the reactor regime. ${ }^{10-12}$ one finds that the lower the collisionality the more important symuetry breaking terms become. Due to the sensitivity of the transport coefficients--in particular the ion thermal conductivity--to asymmetry, reliable methods of evaluation are 
required. Unfortunately, the analytic theory of transport in asymmetric devices has many difficuties particularly when the perturbations produce secondary magnetic wells along the field lines. When secondary magnetic wells are present, the longitudinal invariant $\oint \mathrm{mv}_{\|}$al suffers jumps as particles are collisionlessly trapped and detrapped in the secondary wells.

The Monte Carlo method of evaluating plasma transport coefficients developed in this paper avoids the most serious problem of analytic theory, the need for a simple expression for the particle drift orbits. In Monte Carlo wo:k, the particle drift orbits are exactly evaluated numerically. The Coulomb scattering is included as an appropriate random variation of the ideal constants of the motion.

Related Monte Carlo calculations, but for high energy rather than thermal particles, were made by Lister, Post, and Goldston . 13 Since the work reported in this paper was begun, Monte Carlo evaluations of torsatron transport coefficients were carried out by Potok; Politzer and Lidsky. 14

The Monte Carlo procedure outlined in this paper has several advantages. The use of magnetic coordinates allows one to trivally make the ambipolar electrostractic potential constant on a magnetic surface. In addition the magnetic field can be described by its magnitude alone; so generic features of transport in a plasma geometry can be simply studied. The method of evaluating the diffusion permits a close comparison with analytic theory and the evaluation of the cross terms in the transport equations.

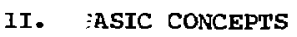

In systems with small asymetries the dominant part of the collision operator is generally pitch angle scattering. ${ }^{2}$ The full collision operator changes both a particle's energy and its pitch. However, neoclassical transport and transport due to asymmetries are primarily caused by the varying 
depth of particle trapping in the magnetic wells which exist along the field iines. Energy scattering does not change the depth of trapping in the magnetic wells, but pitch angle scattering does. There are axceptions to the rule that pitch angle scattering dominates. This occurs, for example, when the transport is dominated by a resonance between the magnetic and electric drifts. 15 The primary emphasis of this paper is on the conceptually simpler case in which pitch angle scattering is dominant. However, a method of inchuding energy scattering will be pointed out.

If the collision operator only scattered particles in pitch, one could define a diffusion coefficient $D(E, \Psi)$ for particles of energy $E$ and radial position $\psi$. The radial position $\psi$ will be defined by the magnetic or constant pressure surfaces. The diffusion equation is

$$
\frac{\partial f}{\partial t}=\frac{1}{s(\psi)} \quad \frac{\partial}{\partial \psi} s \quad D \frac{\partial f}{\partial \psi}
$$

with $f$ the distribution of particles in erer $/$ and $\psi$ space. The function $s(\psi)$ is defined so the volume element of physical space is $\mathbf{d}^{3} \mathbf{x}=s(\psi) d \psi$. Given D, the energy and particle transport coefficients can be evaiuated by taking appropriate moments of the diffusion equation. While doing this evaluation, $f(E, \psi)$ is txeated as a local Maxwellian; so

$$
\frac{1}{f_{m}}\left(\frac{\partial f_{m}}{\partial \psi}\right)_{E}=\frac{1}{n} \frac{d n}{d \psi}+\left(\frac{m v^{2}}{2 T}-\frac{3}{2}\right) \frac{1}{T} \frac{d T}{d \psi}-\frac{e}{T} \frac{d \Phi}{d \psi} .
$$


It should be noted that the moments of $D$ give both the direct and the cross terns in the fluxes.

The diffusion coefficent $D(E, \psi)$ can be evaluated by solving the drift kinetic equation or by Nonte Carlo methods. To relate the method of this paper to other transport calculations, we first consider the evaluation of $D$ from drift kinetic theory. ${ }^{16}$ Let $f=f_{m}(1+\hat{f})$ with $f_{m}$, a local Maxwellian, a function of $\mathrm{E}, \psi$. The deviation of $f$ from a Maxwellian $\hat{f}$ is assumed small. The linearized drift kinetic equation for $\hat{E}$ is

$$
\vec{v}_{d} \cdot \vec{\nabla}_{f}+\left(\vec{v}_{d} \cdot \vec{V}_{\psi}\right) \frac{\partial f_{m}}{\partial \psi}=c_{1}(\hat{E})
$$

with $\mathrm{C}_{1}(\hat{f})$ the linearized collision operator. The drift velocity $\vec{v}_{\mathrm{a}}$ contains the parallel as well as the perpendicular drifts of the particles. A function $h$ is defined which is a solution of

$$
\vec{v}_{d} \cdot \vec{\nabla}_{h}+\vec{v}_{:} \cdot \vec{\nabla}_{\psi} \psi=c_{I}(h),
$$

then approximately

$$
\hat{f} \simeq h \frac{1}{f_{m}} \frac{\partial f}{\partial \psi}
$$


This approximate form for $\hat{\mathfrak{f}}$ involves two approximations in the drift kinetic equation. The first approximation is the neglect of the second term in the expansion

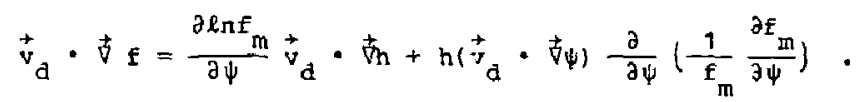

This is just the diffusion approximation, the retention of only lowest order spacial gradients. The second ai roximation is in writing

$$
c_{1}(\hat{f}) \simeq \frac{\partial \ell n f}{a \psi} c_{1}(h)
$$

This approximation would be exact if $c_{1}$ contained only pitch angle scatteing or if $\mathrm{dT} / \mathrm{d} \psi$ were zero. However, the approximation can also be justizied if the transport processes of interest depend only on scattering across small regions of phase space which is in practice the case. Once $\hat{f}$ is written as $n \partial \ln f_{\mathbb{m}} / \partial \psi, D(E, \psi)$ can be defined. Hence, the diffusion function $D(E, \psi)$ exists for all transport processes of interest.

To derive the expression for $D(E, \psi)$ from the drift kinetic theory, the magnetic coordinates $\psi, \theta, \phi_{0}$ must be defined as well as $v, \mu$ velocity coordinates. The magnetic coordinates $\psi, \theta, \phi_{0}$ are defined so $\overrightarrow{\mathrm{B}}=\vec{\nabla}_{\varphi_{0}} \times \vec{\nabla}_{\psi}$ with $\theta$ chosen so the inverse of the coordinate Jacobian $\vec{B} \cdot \vec{\nabla} \theta$ is not zero. The differential element of area of a $\psi$ surface is $\vec{\nabla} \psi$ times the Jacobian, or 


$$
d \vec{S}_{\psi}=\frac{\vec{b} \psi}{\vec{B} \cdot \vec{\nabla} \theta} d \theta d \phi_{0} \text { and } d^{3} x=\frac{d \theta d \phi_{0} d \psi}{\vec{B} \cdot \overrightarrow{7} \theta} \text {. }
$$

For velocity space coordinates, the magnetic moment $\mu$ and velocity $v$ are used. The velocity $\mathrm{v}$ is defined so that the energy is

$$
E=\frac{1}{2} m v^{2}+e \phi_{o}(\psi)
$$

with $\Phi_{0}(\psi)$ the minimum of the electric potential on the surface. (In practice, $\Phi$ is generally cunstant on $\psi$ surface.) The velocity element is then

$$
\mathrm{d}^{3} \mathrm{v}=\frac{4 \pi \mathrm{Bd} \mu \mathrm{v}^{2} \mathrm{dv}}{\mathrm{mv}\left|\mathrm{v}_{\mathrm{u}}\right|}
$$

with $v_{\|}$defined by

$$
\mathrm{E}=\frac{1}{2} \mathrm{mv}_{\mathrm{I}}^{2}+\mu \mathrm{B}+\mathrm{e \Phi}
$$

The total flux of particles across a magnetic surface is then 


$$
\begin{aligned}
& \Gamma_{t}=\int \stackrel{d_{s}^{*}}{\psi} \cdot \int \vec{v}_{d} f d^{3} v \\
& =\int 4 \pi v^{2} d v \int \frac{d \theta d \phi o}{B \cdot+t_{\theta}} \frac{B d \mu}{m v\left|v_{y}\right|}\left\lfloor\left(\vec{v}_{\alpha} \cdot t_{\psi}\right) h \frac{\partial E_{m}}{\partial \psi}\right\rfloor
\end{aligned}
$$

which implies

$$
D(E, \psi)=-\int \frac{\frac{d \theta d \phi_{\theta}}{B \cdot \vec{v}_{\theta}} \frac{B d \mu}{m v / v_{\|}}}{s(\psi)}-\left\{\vec{v}_{d} \cdot \vec{\nabla}_{\psi}\right) \mathrm{h} \text { and } s(\psi)=\int \frac{d \theta d \Phi_{0}}{\vec{B}_{\cdot} \cdot \vec{v}_{\theta}} \text {. }
$$

It is then easy to see that the average particle and heat fluxes are given by

$$
\begin{gathered}
\Gamma=-\int D(E, \psi) \frac{\partial E_{m}}{\partial \psi} 4 \pi v^{2} d v \\
Q=-\int \frac{1}{2} m v^{2} D(E, \psi) \frac{\partial f_{m}}{\partial \psi} 4 \pi v^{2} d v .
\end{gathered}
$$

In Monte Carlo calculations $D$ is determined quite differently. A particle's arift orbit in $\psi, \theta, \phi_{0}$ space is integrated forward in time using $d \psi / d t=\vec{v}_{d} \cdot \vec{\nabla}_{\psi}$, etc. After each time step the pitch angle is changed by an appropriate amount to reproduce the effect of collisions over that time interval. Since collisions axe an ergodic phenomenon, the change in pitch must have a random component. The radial motion of particles is diffusive on long enough time scales which permits an estimate of $D$. 
To develop the Monte Carlo method, consider a large group of particles of given energy which cover a small region in $\psi$ (radial coordinate) space. In the diffusion equation this can be represented by $f(E, \psi)$ being a delta function in energy space and highly localized in $\psi$. Assuming $f$ is normalized so

$$
\int f(E, \psi) s(\psi) d \psi=1, \text { let }\langle\psi\rangle=\int \psi f s d \psi
$$

then

$$
\frac{d}{d t}\langle\psi\rangle=\int \dot{\psi} \frac{\partial f}{\partial t} s d \psi=\left\langle\frac{1}{3} \frac{\partial s D}{\partial \psi}\right\rangle
$$

with two integrations by parts in $\psi$. Similarly

$$
\frac{d}{d t}\left\langle\psi^{2}\right\rangle=2\langle D\rangle+2\left\langle\frac{\psi}{s} \frac{\partial s D}{\partial \psi}\right\rangle
$$

since $\psi$ is high'y localized,

$$
\left\langle\frac{\psi}{s} \frac{\partial s D}{\partial \psi}\right\rangle=\langle\psi\rangle\left\langle\frac{1}{s} \frac{\partial s D}{\partial \psi}\right\rangle
$$

giving 


$$
\langle D\rangle=\frac{1}{2} \frac{\mathrm{d}}{\mathrm{dt}}\left(\left\langle\psi^{2}\right\rangle-\langle\psi\rangle^{2}\right)
$$

This equation requires intepretation. Let $\psi_{j}\left(t_{0}\right)$ be the radial position of the $j^{\text {th }}$ particle after a time $t_{0^{\prime}}$ given that at time zero it was located at $\psi_{0}$. This position $\dot{\psi}_{j}\left(t_{0}\right)$ is determined by following a particle's drift motion with appropriate pitch angle scattering after each time step. Define

$$
D_{j}=\frac{1}{2 t_{a}}\left(\psi_{j}-\dot{v}_{o}\right)^{2} .
$$

The estimate of $D$ from $\mathbf{J}$ particles is

$$
D_{\star}=\frac{1}{\mathbf{J}} \sum_{j}^{J} D_{j}
$$

The statistical distribution of the $D_{j}$ is important, for this allows an es.imate for the number of replications required for $D_{\star}$ to be a good estimate of $D$. To develop the statistics consider the diffusion equation

$$
\frac{\partial g}{\partial t}=D \frac{\partial^{2} g}{\partial x^{2}}
$$

If $g=\delta(x)$ at $t=0$, then after a time $t_{0}$ 


$$
g(x)=\frac{1}{\left(4 \pi D t_{0}\right)^{1} /^{2}} \exp \left(-\frac{x^{2}}{40 t_{0}}-\right) .
$$

The interpretation we wish to place on $g(x)$ is that $g(x) d x$ is the probability a particle which was at $x=0$ at $t=0$ has a position between $x$ and $x+d^{2} x$ at $t=t_{0}$. The estimate of the diffusion coefficient $D_{j}=x^{2} /\left(2 t_{0}\right) ;$ so the probability of $D_{j} l_{Y}$ ing between $D_{j}$ and $D_{j}+D_{j}$ is

$$
g(x) \frac{d x}{d D_{j}} d D_{j}
$$

The probability distribution function for the $D_{j}$ is then

$$
P\left(D_{j}\right)=\frac{1}{\left(8 \pi D D_{j}\right)^{1} / /^{2}} \exp \left(-\frac{D_{j}}{2 D} j .\right.
$$

This is the chi-square distribution with one degree of freedom. Following standard statistical procedures, $D_{*}$ based on $\mathbb{N}$ vaiues of $D_{j}$ lias the Chi-square confidence interval with $\mathrm{N}-1$ degrees of freedom. The $D_{j}$ have a broader

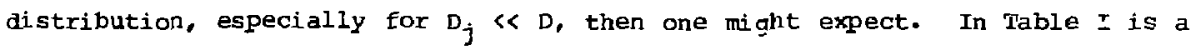
comparison between the Chi-square distribution and the actual distribution of $D_{j} / D_{\star}$ for a run consisting of $450 D_{j}$ 's. 
Table I

\begin{tabular}{ll} 
& $\begin{array}{l}\text { Expected } \\
\text { Cummuiative }\end{array}$ \\
$\mathrm{D}_{\mathrm{j}} / \mathrm{D}$ & Fraction \\
\hline
\end{tabular}

$3.93 \times 10^{-3}$

$1.58 \times 10^{-3}$

0.10

0.45

1.32

2.71

3.84

o
Expected

Number in

$D_{j} / D$ Range.

$22.5 \pm 4.6$

22.5

$67.5 \pm 7.6$

112.5

$112.5 \pm 9.2$

67.5

22.5

$22 \cdot 5$

450. 0 actual

Number in

$D_{j} / S_{*}$ Ránge

30

20

82

121

98

55

18

26

450 
There are several limitations on the Monte Carlo procedure of this paper. First, consider the choice of $t_{0^{\prime}}$ the time between esimates of the diffusion coefficient. Is $t_{0}$ were chusen too long it wowl not adequately represent the time derivative of $\left\langle(\psi-\langle\psi\rangle)^{2}\right\rangle$. This is equivalent to saying $\left|\psi_{j}\left(t_{0}\right)-\psi_{0}\right| / \Psi_{0}$ must be small. If $t_{0}$ were chosen too short then $\psi_{j}\left(t_{0}\right)$ is controlled by deterministic and not randon motion. In other words $\left|\psi_{j}-\psi_{0}\right|$ must be large compared to the radial excursion of a particle executing its drift motion for the process to be diffusive. This limitation can be alleviated by defining

$$
\Psi_{j}\left(t_{0}\right)=j \underbrace{\infty}_{-\infty} 7 \frac{1}{\pi} \exp \left[-\left(\frac{t}{t_{*}}\right)^{2}\right] \psi_{j}\left(t+t_{0}\right) \frac{d t}{t_{*}}
$$

and using $\Psi_{j}\left(t_{0}\right)$ instead of $\psi_{j}\left(t_{0}\right)$ to evaluate $D_{j}$. For this procedure to be valid $t_{*}$ must be short compared to $t_{0^{*}}$ For it to be of any value $t_{\star}$ must be lorg compared to the chafacteristic times of the drift orbits. In practice we ha. - not found it necessary to use $\Psi_{j}\left(t_{0}\right)$ instead of $\psi_{j}\left(t_{0}\right)$. The best prectical yrocedure for choosing $t_{0}$ is apparently to make it greater than or equal to the collision time or the time it rakes a particle to arift around a magnetic surface. That is for $\theta$ and $\phi_{0}$ to increase by $2 \pi$. In plasma devices which can confine particles for mary collision times, the radial excursion after one collision tir.? $\left|\psi_{j}\left(t_{0}\right)-\psi_{0}\right|$ is small compared to the size of the device. Consequently, a . rticular particle can be followed several collision times with several values of $D_{j}$ being determined with 


$$
D_{j}=\frac{1}{2 t_{0}}\left[\psi\left(t_{j}\right)-\psi\left(t_{j-1},\right]^{2} \text {, with } t_{j}=j t_{0}\right. \text {. }
$$

Each time a Monte Carlo run is begun, the particle should be given the same initial values for $\psi$ and energy, but the pitch $v_{\| \prime}, v, e$, and $\phi_{0}$ should be chosen randomly.

A secona limitation of the simple Monte Carlo scheme is the use of a Lorentz scattering operator to change the pitch of the particles. This limitation encompasses not only the lack of energy scattering, which can be easily corrected, but more fundamentally the lack of momentum conservation. If a momentum conserving pitun angle scattering operator is used in orainary drift kinetic theory, the diffusion coefficient $D$ consists of a Lorentz part $D_{1}$ and a momentum conserving part $D_{m}$ (see appendix A of Ref. 2). The Lorentz part $D_{1}$ is given by the Lorentz collision operator which is the scattering of a test particle by a Maxwellian plasma using the Fokker-Planck collision operator. $\because: 9$ other part of the diffusion coefficient $D_{m}$ comes from the motion of the scattering Maxwellian. This motion is required by momentum conservation. If there is at least one symetry direction, one can show momentum conservation "mplies there is no particle transport from like particle collisions. The momentum conserving part of $D, D_{\mathbf{m}}$, exactly cancels the like particle collision contribution of $D_{1}$ to the particle flux. However, in transport due to the breaking of the last symetry jirection, like particle collisions can contribute to particle transport and in analytic theory one finds $D_{\mathrm{m}}=0$. Consequently, the limitation of the Monte Carlo theory described in this paper of Einding $D_{1}$ ratiner than $D$ is not as important as it might first appear. 
Finally, let us consider how energy scattering can be included in the Monte Carlo method. The inclusion of energy scattering as well as pitch angle scatteing presents no problen in the calculation of $\psi_{j}\left(t_{0}\right)$. However, the diffusion coefficient derived from $\psi_{j}\left(t_{0}\right)$ is not $D(E, \psi)$ b.t the ordinary particle diffusion coefficient $D(T, \psi)$

$$
D(T, \psi)=\int_{0}^{\infty} D \mathrm{f}_{\mathrm{m}} 4 \pi \mathrm{v}^{2} \mathrm{dv}
$$

with $f_{m}$ a Maxwellian of temperature $T$. This comes from the energy scattering opęrator containing temperature so that a particle spends time at each energy - in proportion to the Eraction of particles in a Maxwellian at that energyFortunately, one can obtain the various energy moments of $D(E, \Psi)$ from the temperatire dependence of $D(T, \psi)$. To do this it number of Monte Carlo runs are made at different temperatures but with the scattering in energy and pitch held fixed. With the scattering held fixed DiE, $\psi)$ has no temperature dependence so

$$
\mathrm{T}^{2} \frac{\partial D}{\partial \mathrm{T}}=\int \frac{1}{2} \mathrm{mv}^{2} \mathrm{DF} \mathrm{f}_{\mathrm{m}} 4 \pi \mathrm{v}^{2} \mathrm{dv}
$$

'Consequently the derivatives $\partial D / \partial \mathrm{T}$ and $\partial^{2} \not \gamma \partial \mathrm{T}^{2}$ allow one to evaluate the transport coefficients. 
III. DRIFT EQUATIONS IN MAGNETIC COORDINATES

To follow particle drift orbits magnetic coordinates are used. Although these coordinates can be used in arbitrary magnetic fields, ${ }^{17}$ they are simplest for a curl-free field. In this case

$$
\begin{aligned}
\vec{B} & =\vec{\nabla}_{\phi_{0}} \times \vec{\nabla}_{\psi} \\
& =\vec{v}_{X} .
\end{aligned}
$$

To develop an intuitive feeling for $\phi_{0}, \psi$, and $x$ consider the axisymetric tokanak. If $\psi$ is the toroidal flux function, then $\phi_{0}=\phi / q-\theta$ with $\phi$ the toroidal angle, $q(\psi)$ the safety factor, and $\theta$ the poloidal angle. The potential $x=g \phi+I \theta$ witir $g=\mathrm{RB}_{\phi}$ and $I=\mathrm{rB}_{\theta}$ constants in the curl-free case. Within a factor, $g$ and $I$ are the total poloidal and toroidal current. The drift equaticns can be simply derived. The drift velocity is

$$
\vec{v}=v_{B} \frac{\vec{B}}{B}+\frac{m C}{e B}\left(v_{U}^{2}+\frac{1}{2} v_{\perp}^{2}\right) \frac{\vec{B} \times \vec{v} B}{B^{2}}+c \frac{\vec{B} \times \vec{v} \Phi}{B^{2}} .
$$

The equations for the drift orbits are

$$
\frac{d \phi_{0}}{d t}=\dot{v} \cdot \vec{\nabla}_{\phi_{0}}, \frac{d \psi}{d t}=\vec{v} \cdot \vec{\nabla}_{\psi}, \frac{d x}{d t}=\stackrel{+}{v} \cdot \vec{v}_{x} \cdot
$$

To evaluate the terms note 


$$
\begin{aligned}
& \vec{B} \times \vec{\nabla} \phi=\left(\vec{\nabla}_{X}\right) \times\left(\frac{\partial \Phi}{\partial \phi_{0}} \vec{\nabla}_{\phi_{0}}+\frac{\partial \Phi}{\partial \psi} \vec{\nabla}_{\psi}+\frac{\partial \Phi}{\partial \chi} \vec{\nabla}_{X}\right) \\
& \rho_{\|} \equiv v_{\|} \frac{m C}{e B}, \frac{1}{2} m_{\perp}^{2}=\mu B \\
& v_{\|} \stackrel{+}{\mathrm{B} / \mathrm{B}}=\frac{\mathrm{e} \rho_{\mathbb{l}}}{\mathrm{mc}} \vec{\nabla}_{\Phi_{\mathrm{o}}} \stackrel{+}{\times} \nabla \Psi,
\end{aligned}
$$

then

$$
\begin{gathered}
\frac{d \phi_{0}}{d t}=-c \frac{\partial \Phi}{\partial \psi}-\left(\frac{c}{e} \mu+\frac{e B}{m c} \rho_{\|}^{2}\right) \frac{\partial B}{\partial \psi} \\
\frac{d \psi}{d t}=c \frac{\partial \Phi}{\partial \phi_{o}}+\left(\frac{c}{e} \mu+\frac{e B}{m c} \rho_{\|}^{2}\right) \frac{\partial B}{\partial \phi_{o}} \\
\frac{d x}{d t}=\frac{e B}{m c} \rho_{\|} .
\end{gathered}
$$

To obtain an equation for $\rho_{\text {g }}$ use energy conservation

$$
E=\frac{m}{2} v_{i}^{2}+\mu B+e \phi
$$


with the enery $E$ and magnetic moment $\mu$ constants. The sinplest way to proceed is to define a Harriltonian $H \equiv \mathrm{cE} / \mathrm{e}$; so

$$
H\left(\phi_{0}, \psi, x_{,} \rho_{\|}\right)=\frac{1}{2} \frac{e B^{2}}{m c} \rho_{\|}^{2}+\frac{c}{e} \mu B+c \phi .
$$

It is then easy to see that

$$
\frac{d \phi_{o}}{d t}=-\frac{\partial H}{\partial \psi}, \frac{d \psi}{d t}=\frac{\partial H}{\partial \phi_{0}}, \frac{\partial x}{\partial t}=\frac{\partial H}{\partial \rho_{\|}}
$$

energy conservation implies $d H / d t=0$, or

$$
\frac{\partial \rho}{\Omega^{\prime}}=-\frac{\partial H}{\partial x}=-c \frac{\partial \Phi}{\partial x}-\left(\frac{c}{e} \mu+\frac{e B}{m c} \rho_{\|}^{2}\right) \frac{\partial B}{\partial x}
$$

To use these equations cr mputationally, they must be made dimensionless. To do this we use a characteristic magnetic field $B_{0}$ and a system dimension, a, generally the minor radius. With these two scales given, all other quantities can be scaled in the manner of Table II. 
Table II

Physical Quanticy

Frequency

Velocity

Energy

Potentials

Flux

Magnetic Potential

Magnetic Moment
Characteristic Scale

$$
\begin{aligned}
& \omega_{0}=\mathrm{eB}_{0} / \mathrm{mc} \\
& v_{0}=\mathrm{a}_{0} \\
& \mathrm{E}_{0}=\mathrm{m} v_{0}^{2} \\
& \phi_{0}=\mathrm{E}_{0} / \mathrm{e} \\
& \psi_{0}=\mathrm{B}_{0} \mathrm{a}^{2} \\
& x_{0}=\mathrm{B}_{0} \mathrm{a} \\
& \mu_{0}=\mathrm{E}_{0} / \mathrm{B}_{0}
\end{aligned}
$$


with these conventions, the kinetic energy $E_{k}=m v^{2} / 2$ is ijiven by

$\frac{E_{k}}{E_{o}}=\frac{1}{2} \frac{v^{2}}{w_{o}^{2} a^{2}}$;

so $1 /\left(2 E_{k}\right)^{1 / 2}$ in dimensionless units is the number of gyroradi in the system.

To actually use the magnetic coordinates, expressions for $\$$ and $B$ are required. The electrostatic potential is constant within a magnetic surface in scaler pressure are equilibria; so is a function of $\psi$ alone. The Eorm we have used is

$$
\Phi(\psi)=n \frac{E_{k}}{e}\left(1-\frac{\psi}{\psi_{a}}\right)^{2}
$$

with $E_{k}$ the input kinetic energy and $\eta$ a dinensionless constant. The electric iield from this potential vanishes both at the magnetic axis (since $\vec{\nabla} \psi$ vanishes there) and at the plasma edge $\psi=\Psi_{\mathrm{a}}$.

The dependence of the magnetic field strength $B$ on $\phi_{0}, \psi$, and $x$ of course depends on the type dev-ce one is studying. First, consider the axisymetric tokamak. The relations between $\phi_{\mathcal{O}} \psi, x$ and $\psi, \theta, \phi$ imply in the curl free case that

$$
\theta=\frac{1}{1+I / g q}\left(\frac{x}{g q}-\phi_{0}\right) \simeq\left(\frac{x}{g q}-\phi_{0}\right)
$$




$$
\phi=\frac{X}{g}\left(1-\frac{1}{g q / I+1}\right)+\frac{g}{g q / I+1} \cdot \phi_{0}=\underset{g}{x}
$$

where the approximate sign means we have ignored terms of order $I / g q=$ $\left(B_{\theta} / B_{\phi}\right)^{2}$. The toroidal flux

$$
\psi=\frac{1}{2} B_{0} x^{2}
$$

with $B_{0}$ the toroidal field at the magnetic axis. The magnetic field strength in a tokamak is approximately

$$
\begin{aligned}
B & =B_{o}\left[1-\varepsilon_{a}\left(\frac{r}{a}\right) \cos \theta\right] \\
& \simeq B_{o}\left[1-\varepsilon_{a}\left(\frac{\psi}{\psi_{a}}\right)^{1 / 2} \cos \left(\frac{r}{g q}-\phi_{o}\right)\right]
\end{aligned}
$$

with $\varepsilon_{a}$ the inverse aspect ratic at the plasma edge. In dimensionless units

$$
B=1-\varepsilon_{a}(2 \psi)^{1 / 2} \cos \left(\frac{E}{q} x-\alpha\right)
$$

The magnetic field of a stellarator can be derived from the field of a straight helix. ${ }^{18}$ One finds 


$$
\begin{aligned}
B(r, \theta, \phi)=B O & {\left[1-E_{a} \frac{r}{a} \cos \theta-\delta_{a}\left(\frac{r}{a}\right)^{l} \sin (\ell \theta-m \phi)\right.} \\
& \left.-E_{a} \Delta_{a}\left(\frac{r}{a}\right)^{l-1} \cos \theta \sin (\ell \theta-m \phi)\right]
\end{aligned}
$$

with $\varepsilon_{a}$ the inverse aspect ratio, $\delta_{a}$ the helical ripple, and $\Delta_{a}$ the distortion of the magnetic surfaces from circles

$$
\begin{gathered}
\delta_{a}=\frac{\left(m \varepsilon_{a}\right)^{2}}{\ell} \Delta_{a} \\
\Delta_{e}^{2}=\frac{1}{m(l-1) q_{a}}
\end{gathered}
$$

with $q_{a}$ the edge safety factor. One has

$$
q(r)=q_{z}\left(\frac{a}{r}\right)^{2(l-2)} .
$$

Substituting the expressions for $\theta$ and $\phi$ one obtains in dimensionless Eorm 


$$
\begin{aligned}
B=1-\varepsilon_{a}(2 \psi)^{1 / 2} \cos \left(\frac{\varepsilon_{a}}{q} x-\phi_{0}\right)-\delta_{a}(2 \psi)^{\ell / 2} \sin \left[(\ell-m q)-\frac{\varepsilon_{a}}{q} x-\ell \phi_{0}\right\rfloor \\
\\
-\varepsilon_{a} \Delta_{a}(2 \psi)^{l-1 / 2} \cos \left(\frac{\varepsilon_{a}}{q} x-\phi_{0}\right) \sin \left[(l-m q) \frac{\varepsilon_{a}}{q} x-\ell \phi_{0}\right\rfloor
\end{aligned}
$$

with

$$
q=\frac{q_{a}}{(2 \psi)^{l-2}}
$$

To illustrate the evaluation of particle drifts, consider the stellarator. The magnetic field strength in a stellarator (Fi. 45 ) is a complicated function of the location on the magnetic surface (Fig. 1). The variation in field strength along a field line is given by varying $x$ holding $\phi_{0}$ and $\psi$ constant. In Fig. 1 one can see that the variation in field strength along a field Iine consists of a slow oscillation due to the toroidicity and a rapid oscillation due to the hellicity of the stellarator. The toroidal and helical variation in the field strength give rise to two types of trapped particles, toroidal and helically trapped. An individual particle can switch from toroidally trapped to helically trapped and vice versa as a result of the variation in depth and width of the helical wells along its collisionless drift orbit. An example of this behavior is illustrated in Fig. 2. The fact that particles can switch from toroidally to helically trapped implies the longitudinal invariant $J=\mathfrak{f m v}_{\|}$dl is not conserved over the entire drift 
motion. However, the longitudinal invariant is generally conserved while a particle is either helically or toroidally trapped and $J$ conservation can be used to calculate the transition point from helical to toroidal trapping. While carrying out the collisionless drift orbit integrations an 8th order multistep generalization of the Runge-kutta method was used. 1920 The change in energy per time step is a sensitive measure of the accuracy of the numerical integration. For the calculations of $r i g .2$, the ratio of the energy change per time step to the initial energy, $\Delta E / E_{0}$, caused a $20 \%$ adjustment in the time step if it lay outside the range $5 \times 10^{-9}<\Delta E / E_{0}<2.5$ $\times 10^{-8}$. The change in the energy over the entire integration of Fig. 2 was 3 $x 10^{-5}$ the initial energy. The average time step was about $10^{3}$ cyclotron periods.

IV. MONTE CARLO COLLISION OPERATOR

The coll on operatir of primary importance to the Monte Carlo calculations is the Lorentz collision operator

$$
\frac{\partial f}{\partial t}=\frac{\nu d}{2} \frac{\partial}{\partial \lambda}\left(1-\lambda^{2}\right) \frac{\partial f}{\partial \lambda}
$$

with $\lambda=v_{\|} / v$ - The deflection collision Erequency $v_{D}$ is

$$
v_{d}=3\left(\frac{\pi}{2}\right)^{1 / 2} v_{B} \frac{\Phi(x)-\Psi(x)}{x^{3}}
$$

with $v_{B}$ the Braginskii collision frequency 


$$
v_{B}=\frac{4}{3}\left(\frac{\pi}{m}\right)^{1 / 2} \frac{\Lambda e^{4} n}{T^{3 / 2}}=\frac{\Lambda / 10}{3 \times 10^{6}}\left(\frac{2}{A}\right)^{1 / 2} \frac{n}{T^{3 / 2}} .
$$

In this formila $\Lambda$ is the Coulomb logarithm, $A$ the atomic mass of the ions, $n$ the electron density in particles per $\mathrm{cm}^{3}$, and $\mathrm{T}$ the temperature in electron volts. For $\mathrm{n}=10^{14} / \mathrm{cm}^{3}$ and $\mathrm{T}=10^{4} \mathrm{ev}$, the Coulomb logarithm is $\Lambda=18.4$. The variable $x=v /(2 T / m)^{1 / 2}$ with $v$ the particle velocity. The functions $\Phi$ and I' are given by

$$
\begin{aligned}
& \Phi(x)=\frac{2}{\sqrt{\pi}} \int_{0}^{x} e^{-t^{2}} d t \\
& \Psi(x)=\frac{\Phi-x \Phi^{-}}{2 x^{2}}
\end{aligned}
$$

The thermal deflection rate is defined by $v_{d}(x=1)=2.3646 v_{B}{ }^{*}$ The dimensionless collision frequency is $v_{\alpha^{\prime}} \omega_{c}$ or

$$
\nu_{d}(x=1)=3.03 \times 10^{-10} \frac{n}{B T^{3 / 2}}\left(\frac{A}{2}\right)^{1 / 2}
$$

with $B$ is Gauss using $\Lambda=18.4$.

Shanny, Dawson, and Greene 21 as well as Lister, et al. ${ }^{13}$ have given a Monte Carlo equivalent of the Lorentz operator based on the Gaussian distribution. However, we used a simpler Monte Carlo equivalent operator based on the binomial distribution. To derive this operator, let 


$$
\langle\lambda\rangle \equiv \int_{-1}^{1} \lambda \mp d \lambda
$$

ther one can show by integrating by parts that

$$
\frac{d\langle\lambda\rangle}{d t}=-v_{1}\langle\lambda\rangle, \frac{d\left\langle\lambda^{2}\right\rangle}{d t}=v_{d}\left(1-3\left\langle\lambda^{2}\right\rangle\right) .
$$

The square of the standard deviation of $f$ in pitch space

$$
0^{2}=\left\langle\lambda^{2}\right\rangle-\langle\lambda\rangle^{2}
$$

broadens in time with

$$
\frac{d \sigma^{2}}{d t}=\nu_{d}\left(1-3\left\langle\lambda^{2}\right\rangle+2\langle\lambda\rangle^{2}\right)
$$

Suppose at $t=0$, $f$ were a delta function alout $\lambda=\lambda_{0^{*}}$ At that time

$$
\frac{d\langle\lambda\rangle}{d t}=-v_{d} \lambda_{0}, \frac{d \sigma^{2}}{d t}=v_{d}\left(1-\lambda_{0}^{2}\right) .
$$


After a short time $t$, we expect $f$ to be a Gaussian centered at $\lambda=\lambda_{0}\left(1-\nu_{d} t\right)$ with standart deviation $\left[\left(1-\lambda_{0}{ }^{2}\right) v_{a}\right]^{1 / 2}$. Suppose we thought of the broadening of $f$ as being due to a large number of steps in pitch of equal size but of random sign. The distribution function for obtaining $m$ plus values in n trials with equal probability for plus and minus, the binomial distribution, is

$$
P(m)=\frac{1}{2^{n}} \frac{n !}{m !(n-m) !} \text {. }
$$

Let $j$ be the number of pluses ainus the number of miruses, $j=2 \mathrm{~m}-\mathrm{n}$, then for $n>1$,

$$
P(j)=\frac{1}{(2 \pi n)^{1 / 2}} \text { exp }\left\lfloor-\frac{j^{2}}{2 n}\right\rfloor \text {. }
$$

The standard deviation of $j$ is $/ n$. To reproduce the standard deviation 0 , the $\lambda$ steps must each have a magnitude $\left.1\left(1-\lambda_{0}\right)^{2} \nu_{d}\right]^{l} /^{2}$ with $\tau$ the length of time between the steps. After $n$ steps the time $t=\boldsymbol{n} \tau$, and the standard deviation of the $\lambda$ distribution will be the step size times $/ n$, or

$$
\sigma=\left[\left(1-\lambda_{0}\right)^{2} v_{D} t\right]^{1 / 2}
$$


This agrees with our earlier expression for $\sigma$. Clearly if the pitch is changed from $\lambda_{0}$ to $\lambda_{n}$ after a time step of length $\tau$ with

$$
\lambda_{n}=\lambda_{0}\left(1-\nu_{d} \tau\right) \pm\left\lfloor\left(1-\lambda_{0}\right)^{2} \nu_{d} \tau\right]^{1 / 2}
$$

then the effects of the Lorentz scattering operator will be reproduced fur $\nu_{\mathrm{d}^{T}} \ll 1$. The symbol \pm means the sign is to be chosen randomly, but with equal probability for plus and minus.

The operator of Eq. 58 has the important feature that if $\left|\lambda_{0}\right|<1$, then $\left|\lambda_{n}\right|<1$. It is physically obvious that the pitch must be less than unity, however, an otherwise valid Monte carlo operator could allow $\lambda$ to go out of this range orovided the amount $|\lambda|$ exceeded unity went to zero as $V T$ went to zero. To show $\left|\lambda_{n}\right|<1$, let us assune for simplicity $v_{D}^{T}$ is small compared to unity. clearly, $\left|\lambda_{0}\right|$ must be near one for a problem to occur; so we let $\lambda_{0}=1-\delta$ with $\delta \ll 1$. The largest $\lambda_{n}$ can be is

$$
\lambda_{n}=1-\left(\delta+v_{d} \tau\right)+\left(2 \delta v_{d} \tau\right)^{1 / 2}
$$

The maximum value of $\lambda_{n}$ as $\delta$ is varied occurs at $\delta=v_{d} \tau / 2$; so

$$
\left|\lambda_{n}\right|<1-\frac{v_{d} \tau}{2}
$$


To have a good representation of the Lorentz operator, $v_{d} \tau$ must clearly be mich less than one. It is easy to see that $\left(v_{d}\right)^{1 / 2}$ significantly smaller than the smallest regions of interest. in $\lambda$ space is the actual requirement. In the Monte Carlo transport calculations, the time step of the integrations, $\tau$, is chosen th be sinall enough so the orbit equatiors are energy conserving. This choice insures several time steps while a particle crosses any regime over which the magnetic field strength changes significantly. This choice also insures there are several scatters before a particle has changed its pitch significantly.

To illustrate the action of the Monte Carlo Lorentz scattering operator we evaluated the fraction of the time a particle spent at each pitch while subjected to the scattering operator. In Fig. 3 this fraction is plotted at three points in time for a particle which started with zero pitch. After many collision times, the paxticle has spent equal time at each pitch. The fluctuations in time spent are of order $1 /\left(v_{d} t\right)^{1 / 2}$. While carrying out this calculation the time step was $2 \times 10^{-4} / v_{\mathrm{d}}$.

The energy scattering equivalent of the Lorentz scattering operator is

$$
\frac{\partial f}{\partial t}=\frac{1}{v^{2}} \frac{\partial}{\partial v}\left[v^{2} v_{E}\left(v f+\frac{T}{m} \frac{\partial f}{\partial v}\right)\right]
$$

with

$$
\nu_{E}=3\left(\frac{\pi}{2}\right)^{1 / 2} v_{B} \frac{\Psi(x)}{x} .
$$


This energy scattering operator can be derived irom the Fokker-Planck collision operator by assuming particles scatter on an background Maxwellian of temperature $T$. One can show that the energy scattering operator causes any distribution function $f$ to relax to a Maxwellian, $f_{\mathfrak{m}^{\prime}}$ of temperature $T$, by an $H$ theorem. The role of $\mathrm{H}$ is played by

$$
H=\int_{0}^{\infty}\left(\frac{1}{2} \pi v^{2}\right) f 4 \pi v^{2} d v
$$

A Monte-Carlo equivalent energy scattering operator can be found by evaluating

$$
\frac{d}{d t}\langle E\rangle=\frac{d}{d t} \int_{0}^{\infty}\left(\frac{1}{2} m v^{2}\right) \text { f } 4 \pi v^{2} d v
$$

and $d\left\langle E^{2}\right\rangle / d t$. This operator is

$$
E_{n}=E_{0}-\left(2 \nu_{E} \tau\right)\left[E_{0}-\left(\frac{3}{2}+\frac{d \ell n v_{E}}{d l_{n} E}\right) T\right] 2\left[T E_{O}\left(\nu_{E} \tau\right)\right]^{1 / 2}
$$

One can show the energy can never go negative. That is it if $E_{0}>0$, then $E_{n}>0$. 
In Fig. 4 it is demonstrated that a partỉcle which started with thermal energy spends time at various energies in proportion to the Maxwellian distribution. If $n(E)$ is the fraction of time a particle has energy $E$ then

$$
n(E) / \sqrt{ } E \propto \exp (-E / T)
$$

is the expected distribution.

\section{EVALUATION OF D FOR A STELLARATOR}

To illustrate the power of the Monte Carlo method, the problem of ion transport in a stellarator was studied. Analytic evaluations of ion heat transport in stellarators have given pessimistic results.16,22 At the time the calculations reported in this paper were undertaken no Monte Carlo evaluations of transport in stellarators had been carried out. However, more recent Monte Carlo work has given collaborative results. ${ }^{14}$

While evaluating the diffusion coefficient a fourth order accurate KuttaMerson process was used for the orbit integrations. After each orbit time s'iep the pitch was changed by using the Monte carlo, Lorentz collision operator. It was found that a slight improvement in the representation of collisional effects occured if the Monte Carlo scattering operator was called several times rather than just onite at the end of a time step. That is if the time step was $\tau_{0}$ the Monte Carlo scattering operator was called $j$ times with $\tau=\tau_{o} / j \cdot$ In practice we used $j=5$.

The stellarator configuration studied was that of Figs. 2 and $3, z=2$, $m=6$, and $\varepsilon=1 / 7$. The transport coefficients were studied half way out in toroidal flux from the center $(r=a / \sqrt{2})$ with the local safety factor assumed 
to be two. Figure 5 gives the time history of a particle's motion for six collision times $\left(6 / v_{d}\right)$. The dependence of the diffusion coefficient $D$ on collisionality was studied both with and without a radial electric field. In Fig. 6 one can see there is little dependence of $D$ on collisionality contrary to early analytic predictions. However, at a fixed collisionality D was found to depend quadratically on kinetic energy.

For comparison purposes and to test the code, D was evaluated for a tokamak with the same safety factor aud aspect ratio as the stellarator. The low collisionality value for $D$ in a tokamak can be easily evaluated a:alytically to lowest order in the inverse aspect ratio (see Appendix A, Ref. 2). This is the neo-classical banana regime. In dimensionless form the analytic result for a Lorentz collision operator is

$$
D=[2 \varepsilon(\psi)]^{1 / 2} \frac{\sigma}{E_{a}^{2}} q^{2} E_{K} v_{d}
$$

The local aspect ratio is $\varepsilon(\psi), \sigma=0.689 .$. . is a numerical constant, $E_{a}$ is the edge aspect ratio, and $E_{k}$ is the kinetic energy As can be seen in Fig. 6 the tokamak results of the Monte Carlo code agree with the analytic formula at low collisionality. At higher collisionality, the code results demonstrate the bending over of $D$ into the so-called plateau regime.

ACKNOWLEDGMENTS

The work was supported by the United States Department of Energy, Contract Number DE-ACO2-76-CHO 3073. 
1A. A. Galeev and R. J. Sagdeev, Zh. Eksp. Teor, Fi. 53 (1967) 348 [Sov. Phys. JETP, 26, (1968) 233]

2M. N. Rosenbluth, R. D. hazeltine and F. L. Hinton, Phys. Fluids 15 (1972) 116.

3F. L. Hinton and R.D. Hazeltine, Rev. Mod. Phys. 48 (1976) 239.

4A. Pytte and A. H. Boozer, Report PPPI-1552, Plasma Physics Laboratory, Princeton University (1979).

${ }^{5}$. W. Kerst, J. Nucl, Energy, Pt.C 4 (1962) 253.

$6_{\mathrm{H}}$. Grad, Phys. Fluids 10 (1967) 137.

7M. N. Rosenbluth, R. Z. Sagdeev, J. B. Taylor and G. M. Zaslavski, Nucl. Fusion 6 (1966) 297.

BN. N. Filonenko, R. Z. Sagdeev, and G. M. Zaslavsky, Nucl. Fusion 7 (1967) 253.

9. H. Boozer and A. B. Rechester, Phys. Fluids 21 (1978) 682.

10 T. E. Stringer, Nucl. Fusion 12 (1972) 689.

$11 \mathrm{~J}$. W. Connor and R. ․ Hastie, Nucl. Fusion 13 (1973) 221.

12A. H. Boozer, Report PPPL-1619, Plasina Physics Laboratory, (Jan. 1980). To be published in Phys. Fluids.

${ }^{13} \mathrm{G}$. G. Lister, D. E. Post, and R. Goldston in the "Symposium on Plasma Heating in Toroidal Devices, 3rd", (Varenna, Italy 1976), see p. 303.

${ }^{14}$ R. E. Potok, P. A. Politzer, and L. M. Lidsky, to be published.

15 E. F. Jaeger, C. L. Hedrick, and D. A. Spong. Nucl. Fusion 19, (1979) 1627.

16E. A. Frieman, Phys. Fluids 13 (1970) 490 .

17 A. H. Boozer, Phys. Fluids 23 (1980) 904. 
18 A. I. Morozov, L. S. Solovev in Reviews of Plasma Physics, Vol. 2 (M. A. Leontovich, Ed) Consultants Bureau, N. Y. (1966), see p. 1.

19 T. J. Martin, private communication.

$20 \mathrm{~J}$. C. Butcher, J.A.C.M., 14 (1967) 84 .

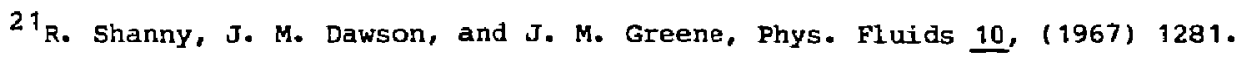

22J. W. Connor and R. J. Hastie, Phys. Fluids 17 (1974) 114. 


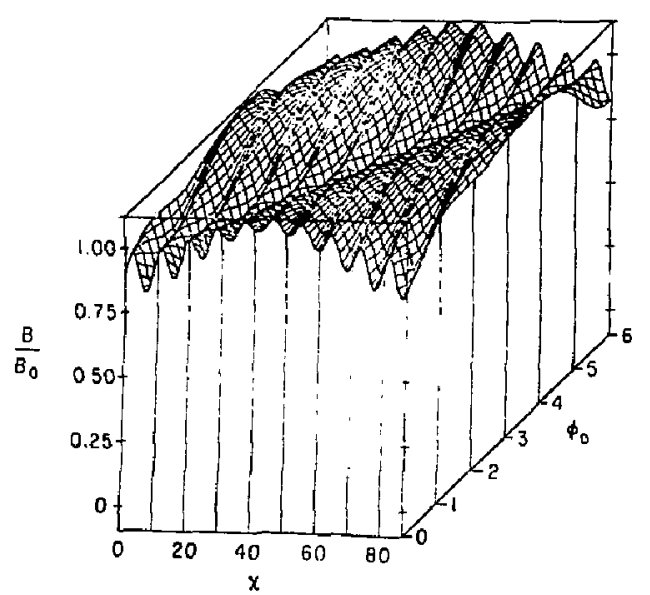

(PPPL-802225)

Fig. 1. Stellarator Field Strength. The strength of the stellarator field in a given magnetic surface (half way out in $f(u x)$ is plotted versus $x$, the variable along the 1 ines, and $\phi_{0}$, the variable across the lines, for one period in each direction. The slow variation in the $\phi_{0}$ and $y$ directions is due to torodicity while the rapid oscillation is due to helicity.

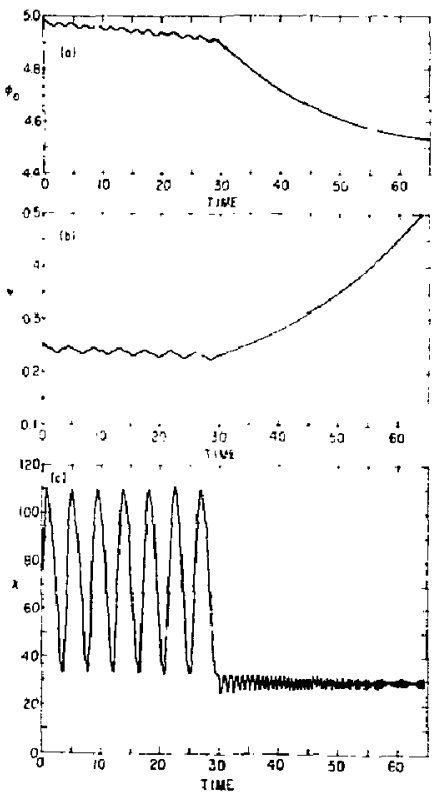

(PPPL-802228)

Fig. 2. Collisionless Stellarator Particle Orbit. The time dependence of $\phi_{0}, \psi$ and $x$ is illustrated for a particle moving in the stellarator field. As can be seen from $x$, the coordinate along the field lines, the particle switches from being trapped in a toroidal well to a ripple well as a result of its collisionless drift motion. The time units are $10^{5} / \omega_{c}$. 

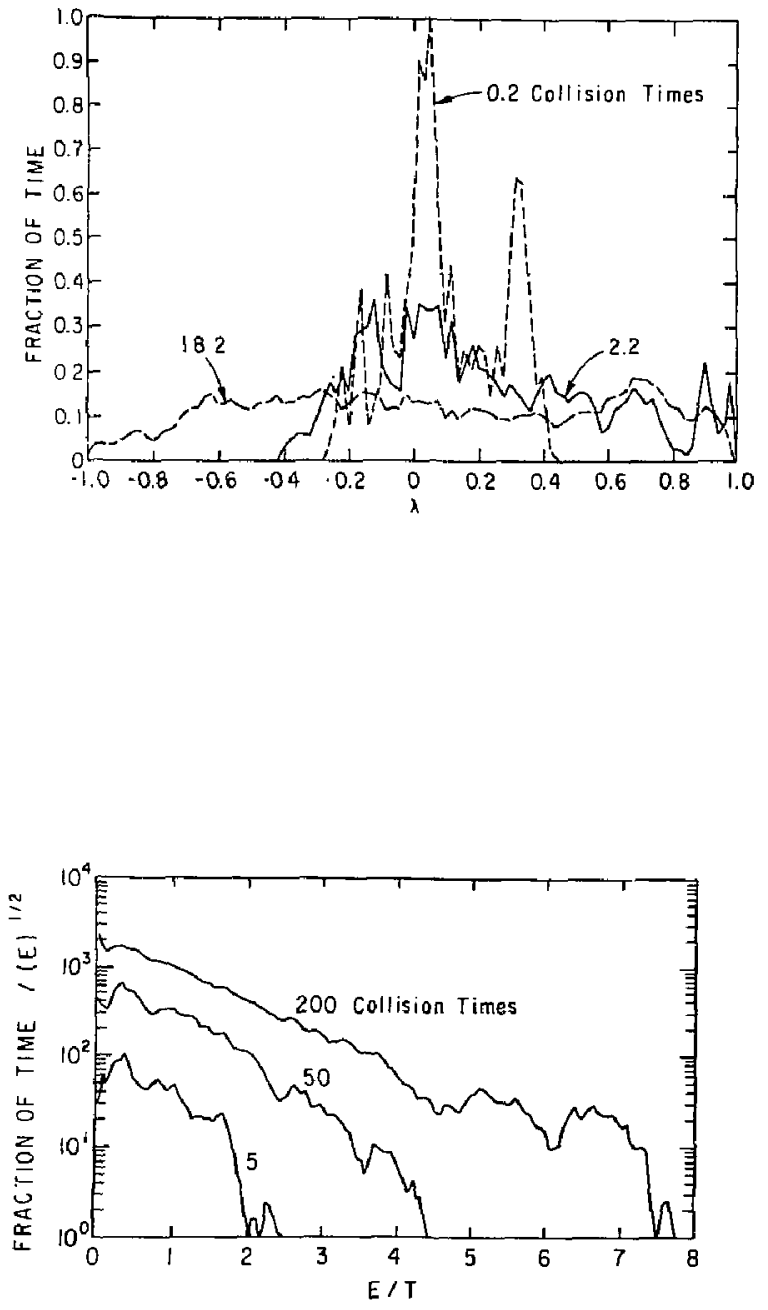

(PPPL-802226)

Fig. 3. Relaxation in Pitch Space. A particle started with $\lambda=v_{\|} / v=0$ was followed with the Monte Carlo equivalent of the Lorentz collision operator. Plotted is the time the particle has spent at various values of pitch, $\lambda$, after $0.2,2.2$, and 18.2 collision times. The curves are normalized so there is equal area under each. After many collision times, the particle has spent equal time at all values of the pitch between $\lambda=+1$ and $\lambda=-1$.
(PPPL-802227)

Fig. 4. Relaxation in Energy Space. A particle started with the thermal energy $E=T$ was followed with the Monte Carlo equivalent of the energy scattering operator. The vertical scale is in arbitrary units but proportional to the fraction of time a particle has spent at each value of the energy divided by the square root of energy. A Maxwellian would give a straight Iine. 
Fig. 5. Collisional stellarator Particle Motion. The time dependence of $\phi_{0}, \psi$, and $X$ is illustrated for a particle moving in the stellarator field for 6 collision times. The larger jumps in $\psi$ occur for trapped particles. This is demonstrated in the figures by large jumps in $\psi$ occuring at the places where $d x / d t=0$. The time units are $10^{6} / \mathrm{s}_{\mathrm{c}}$.

(PPPL-802244)

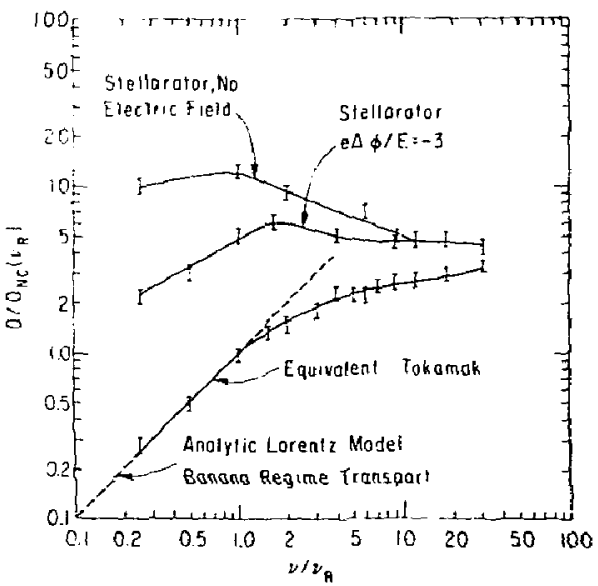

Fig. 6. Diffusion coefficient in a Stellarator. The diffusion coefficient $D(E, \psi)$ is plotted for a thermal particle in a stellarator with and without on ambipolar electric field and in an equivalent tokamak. The reactor collision frequency $v_{R}$ is defined by $n=10^{14} / \mathrm{cm}^{3}$, $I=10 \mathrm{kev}, \mathrm{B}=50 \mathrm{kG}$. The symbol $D_{N C}\left(v_{R}\right)$ means the value of the neoclassical diffusion at $v=v_{R}$. The parameters of the stellarator were $\ell=2, m=6, \epsilon=1 / 7$, and $\mathrm{q}=2$. 